\title{
Efficiently parallelized modeling of tightly focused, large bandwidth laser pulses
}

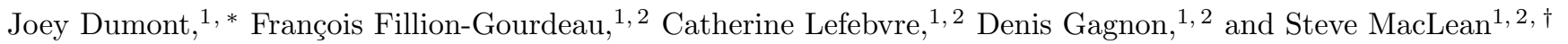 \\ ${ }^{1}$ Université du Québec, INRS-Énergie, Matériaux et Télécommunications, Varennes, Québec, Canada, J3X 1 S2 \\ ${ }^{2}$ Institute for Quantum Computing, University of Waterloo, Waterloo, Ontario, Canada, N2L 3G1
}

(Dated: November 15, 2018)

\begin{abstract}
The Stratton-Chu integral representation of electromagnetic fields is used to study the spatiotemporal properties of large bandwidth laser pulses focused by high numerical aperture mirrors. We review the formal aspects of the derivation of diffraction integrals from the Stratton-Chu representation and discuss the use of the Hadamard finite part in the derivation of the physical optics approximation. By analyzing the formulation we show that, for the specific case of a parabolic mirror, the integrands involved in the description of the reflected field near the focal spot do not possess the strong oscillations characteristic of diffraction integrals. Consequently, the integrals can be evaluated with simple and efficient quadrature methods rather than with specialized, more costly approaches. We report on the development of an efficiently parallelized algorithm that evaluates the Stratton-Chu diffraction integrals for incident fields of arbitrary temporal and spatial dependence. This method has the advantage that its input is the unfocused field coming from the laser chain, which is experimentally known with high accuracy. We use our method to show that the reflection of a linearly polarized Gaussian beam of femtosecond duration off a high numerical aperture parabolic mirror induces ellipticity in the dominant field components and generates strong longitudinal components. We also estimate that future high-power laser facilities may reach intensities of $10^{24} \mathrm{~W} / \mathrm{cm}^{2}$.
\end{abstract}

\section{INTRODUCTION}

Recent proposals for high-power laser infrastructures such as ELI and APOLLON [1, 2] have opened the possibility of an experimental detection of strong-field quantum electrodynamics (SF-QED) effects including vacuum polarization, Breit-Wheeler pair production, nonlinear Compton scattering and Schwinger pair production [3] The high intensities required to observe these effects can be approached by using a temporally compressed highpower laser in conjunction with a tight focusing scheme [8, 9].

In the tight focusing regime, the spatial extension of the field is on the same order of magnitude as its wavelength and, as a consequence, paraxial fields fail to properly model the spatio-temporal properties of the field at the focal spot [10, 11. This approach generally considers an expansion valid only for small diffraction angles $\epsilon \ll 1$, where $\epsilon=\lambda / \pi w_{0}$ is the ratio of the wavelength of the beam $\lambda$ to its transverse width $w_{0}$. Tightly focused beams are characterized by $\epsilon \gg 1$ and, in addition, any realistic analysis in this regime must retain the vector nature of the fields. Moreover, to properly represent the experimental reality, the effect of the reflection on the mirror should be considered, requiring a formulation that properly accounts for the boundary conditions on the reflecting surface.

Methods that consider the vector character of the fields have been used before, starting with Ignatowski's study of Maxwell's equations in parabolic cylindrical coordinates [12, 13. Later, Kottler [14, 15] formulated the

* Corresponding author: joey.dumont@gmail.com

$\dagger$ steve.maclean@emt.inrs.ca reflection problem as an integral equation using Green's function techniques. Stratton and Chu [16] then generalized Kottler's result and streamlined its derivation. Richards and Wolf 17, 18 constructed a different integral technique based on energy conservation and the farfield approximation. The Stratton-Chu and RichardsWolf methods have been used to study tight focusing geometries in multiple recent publications [19 26]. Almost all of these articles have considered simplifications such as monochromatic light or plane wave incidence, or both, even though they are not inherent to the Stratton-Chu and Richards-Wolf methods.

Other studies have used a completely different approach and solved Maxwell's equations with specific source geometries (e.g. complex source beams) [27, 28, or given four-potential $A_{\mu}$ [29] in order to model the tight focusing regime. These analytical results are extremely useful in that they provide closed-form approximations to tightly focused fields. However, they fail to capture the details of the electromagnetic fields generated by the reflection off a given mirror geometry, as we will show.

In this article, we report on a numerical implementation of the Stratton-Chu diffraction integrals that fully models the reflection of temporally short fields off high numerical aperture optical systems. We review the derivation of the Stratton-Chu equations, noting that the only approximation used to model the reflection problem is the physical optics approximation. This approximation does not require any assumption on the spatial or temporal dependence of the incident field and is valid for almost any mirror shape. Our specific implementation is thus able to model the reflection of an experimentally realistic beam profile with a complex broadband spectrum characteristic of the short pulses produced in high-power laser systems [2]. In practical terms, the Stratton-Chu in- 
tegrals take as an input the laser field impinging on the focusing mirror and return the focused field at any point in space and time. This formalism is thus useful to study tightly focused fields, as the unfocused field coming from the laser chain is typically known to a high experimental accuracy, while the reflected, tightly focused field is not, due to the difficulty of imaging very small focal volumes at high intensities.

By construction, the integral representation decouples the regions in which the field is computed from the region on which we perform the numerical work, which in our case consists of quadratures. To compute the field in the vicinity of the focal spot of a given mirror, it is therefore not necessary to model the propagation of the field at every point between the mirror and the observation point. This confers a distinct numerical advantage to the integral method compared to the finite-difference time-domain (FDTD), finite-difference frequency-domain (FDFD) and even traditional finite element methods (FEM). These latter methods typically discretize the whole region of space that contains the mirror and the focal spot. This is especially taxing in the tight focusing regime where the focal spot must be discretized with sub- $\lambda$ resolution, while the mirror is several orders of magnitude larger than $\lambda$. Indeed, laser beams in the optical region of the spectrum have $\lambda \sim 1 \mu \mathrm{m}$ while the mirror has an aperture in the centimeter range. This results in a spatial mesh so fine that it would require unmanageable amounts of memory to accommodate, even on a modern supercomputer. A crude estimate using a Yee lattice yields a memory requirement approaching the exabyte ( $10^{6}$ terabytes). This can be mitigated in FEM techniques by using variable mesh sizes, but this does not remove the inherent issue that the whole space must be discretized. Moreover, the FDTD, FDFD and FEM techniques must use an artificial boundary, such as perfectly matched layers [30, to account for the free-space propagation after the reflection on the mirror without meshing the whole space.

Despite its advantages, the versatility of the integral method comes at a cost. By estimating the number of floating point operations needed to evaluate the reflected field in a $\lambda^{3}$ volume around the focal point when a broadband pulse impinges on a mirror, we can estimate a runtime of several weeks on a single modern CPU core. Furthermore, even though the memory requirements are lessened compared to standard methods, they can still be prohibitive. Fortunately, the integrals can be evaluated in parallel very efficiently. This allows for a manageable runtime, ranging from a few hours to a few days depending on the parameters of the incident beam, and greatly diminishes the memory required of each CPU core.

The accurate characterization of electromagnetic fields in the strong focusing regime is essential in the context of the experimental observation of SF-QED effects. While most studies use simple models, such as plane or paraxial waves, to describe the laser field while computing SFQED observables, recent studies have shown that the spatial structure of the field can alter the observables related to pair production [3, 31, 32, vacuum wave mixing [7, 33] and nonlinear Compton scattering [34 in a non-trivial way. Moreover, analytical tools recently developed by Di Piazza facilitate the study of the effects of tightly focused fields on SF-QED observables [35-37. Tightly focused fields have also found use in practical applications, such as direct electron acceleration [38 40], microscopy [20, 41] and plasma physics modeling [42.

The article is structured as follows. Section [I contains a detailed review of the Stratton-Chu representation and their reduction to a set of diffraction integrals. The physical optics approximation is introduced and shown to arise from the first term in the Liouville-Neumann expansion. Section III discusses practical details of the implementation, such as the spatial and temporal discretization schemes used, and describes the efficient parallelization of the algorithm with the domain decomposition method. It also shows that, for a parabolic mirror, the integrands of the Stratton-Chu equations do not possess the strong oscillations typical of diffraction integrals, thus simplifying their evaluation. Section IV validates the numerical implementation and analyzes the fields computed via the Stratton-Chu equations and shows that the geometry of the reflecting surface should be taken into account. Our results show that future high-power laser facilities could reach the record intensity of $10^{24} \mathrm{~W} / \mathrm{cm}^{2}$, notwithstanding imperfect vacua and quantum effects. We conclude in Section V.

\section{STRATTON-CHU DIFFRACTION}

This section sets up the theoretical apparatus that will be used to model the reflection of short optical laser pulses off high numerical aperture (strongly focusing) mirrors. Starting from Sancer's form of the Stratton-Chu integral representation 43, we derive a set of hypersingular integral equations that describe the fictitious currents impressed on the mirror by the incident field. We show that the hypersingularity can be attributed to the openness of the mathematical surface that represents the mirror, as it reduces to a singular integral in the case of a closed surface. Then, using the fact that the resulting integral operator is compact, we conclude that the physical optics approximation is recovered in the first term of the Liouville-Neumann expansion. Throughout this section, we use Lorentz-Heaviside units in addition to setting the speed of light to unity, i.e. $c=1$.

The Stratton-Chu representation has been derived using a variety of techniques [15, 16, 44, 45], but perhaps the most elegant derivation comes from Sancer [43]. For the purposes of this work, it suffices to say that the derivation is based on the application of several vector and tensor calculus identities and the dyadic-vector version of Green's theorem [46] on Maxwell's equations. The geometry of the problem is defined as follows. Sources of electric and magnetic currents, whose support are $V_{e}$ and 


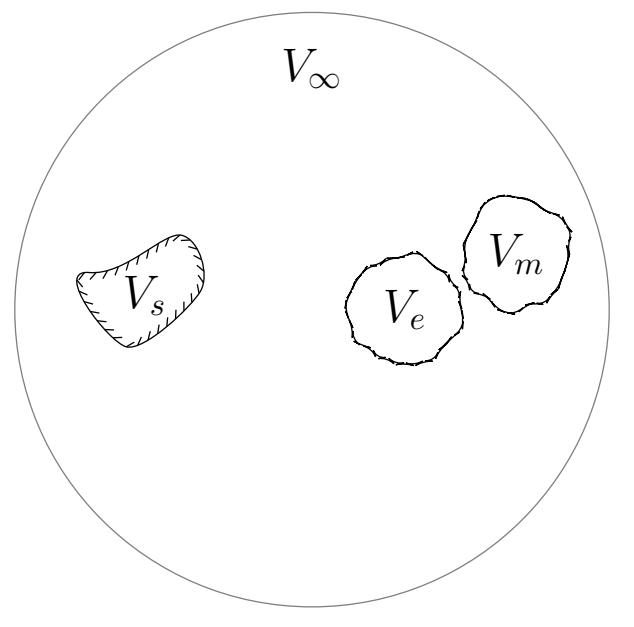

FIG. 1. Scattering system used in the derivation of the Stratton-Chu representation. $V_{e}$ and $V_{m}$ are the support of the electric and magnetic sources, respectively, while $V_{s}$ is the scattering object. The volume $V_{\infty}$ is a sphere whose radius eventually goes to infinity and contains $V_{e}$ and $V_{m}$, but not $V_{s}$.

$V_{m}$, are supposed to exist in free space, represented by $V_{\infty}$ (Fig. 11). Mathematically speaking, both $V_{e}$ and $V_{m}$ are subsets of $V_{\infty}$ and can overlap, and $V_{\infty}=\mathbb{R}^{3} \backslash V_{s}$ is all of free space save from the volume occupied by the scatterer. Applying the relevant theorems in this particular geometry yields a representation in terms of integrals over the surface of the scatterer $S=\partial V_{s}$. The representation is valid for surfaces of arbitrary shape, including open surfaces. The material properties of the scatterer are taken into account by enforcing proper boundary conditions. The representation, for a monochromatic field, reads 43 ]

$$
\begin{aligned}
\boldsymbol{E}^{\prime}=\boldsymbol{E}_{\mathrm{inc}}^{\prime}+ & \iint_{S}\{i k(\hat{\boldsymbol{n}} \times \boldsymbol{B}) g+(\hat{\boldsymbol{n}} \times \boldsymbol{E}) \times \nabla g \\
& \left.+\frac{i}{k} \nabla \nabla g \cdot(\hat{\boldsymbol{n}} \times \boldsymbol{B})\right\} d S, \\
\boldsymbol{B}^{\prime}=\boldsymbol{B}_{\mathrm{inc}}^{\prime}+ & \iint_{S}\{-i k(\hat{\boldsymbol{n}} \times \boldsymbol{E}) g+(\hat{\boldsymbol{n}} \times \boldsymbol{B}) \times \nabla g \\
& \left.-\frac{i}{k} \nabla \nabla g \cdot(\hat{\boldsymbol{n}} \times \boldsymbol{E})\right\} d S,
\end{aligned}
$$

valid $\forall \boldsymbol{r}^{\prime} \in V_{\infty}$. In these equations, $\left\{\boldsymbol{E}_{\text {inc }}, \boldsymbol{B}_{\text {inc }}\right\}$ are the incident fields and are assumed to arise from the sources and currents in $V_{e}$ and $V_{m}$, respectively. We thus only require that the incident fields are solutions of Maxwell's equations. The primed coordinates represent any point in $V_{\infty}$, while the unprimed coordinates represent points on $S$, and are integrated over. $\left\{\boldsymbol{E}^{\prime}, \boldsymbol{B}^{\prime}\right\}$ thus represent the field at any given point in $V_{\infty}$, while $\{\boldsymbol{E}, \boldsymbol{B}\}$ denote the field on the surface $S$. The notation $\boldsymbol{E}^{\prime}[\boldsymbol{E}]$ is short for $\boldsymbol{E}\left(\boldsymbol{r}^{\prime}, k\right)[\boldsymbol{E}(\boldsymbol{r}, k)]$ where $k=2 \pi / \lambda$ is the wavenumber of the laser pulse. $g$ is the scalar Green's function

$$
g\left(\boldsymbol{r}, \boldsymbol{r}^{\prime}\right)=\frac{e^{i k\left|\boldsymbol{r}-\boldsymbol{r}^{\prime}\right|}}{4 \pi\left|\boldsymbol{r}-\boldsymbol{r}^{\prime}\right|} .
$$

It is the only factor in Eq. (1) that depends on both the integration variables $\boldsymbol{r}$ and the observation variables $\boldsymbol{r}^{\prime}$.

Equation (1) is an integral representation that expresses the field at any point in $V_{\infty}$ as integrals of the (yet unknown) fields $\{\boldsymbol{E}, \boldsymbol{B}\}$ over the surface of the scatterer. To find the value of the fields on the scatterer, we take the limit $\boldsymbol{r}^{\prime} \rightarrow S$. This results in an integral equation on $S$, which can be solved iteratively. However, care must be taken in the evaluation of this limit as $g$ has a simple pole at $\boldsymbol{r}=\boldsymbol{r}^{\prime}$, i.e. on the surface of the mirror. This singularity is dealt with analytically by deforming the surface at $\boldsymbol{r}=\boldsymbol{r}^{\prime}$ to a hemispherical surface with vanishing radius $R$. Let this contour be $S_{\epsilon}$. The surface integrals can now be written as

$$
\lim _{\boldsymbol{r}^{\prime} \rightarrow S} \iint_{S}\{\cdot\} d S=\lim _{R \rightarrow 0}\left(\iint_{S / S_{\epsilon}}+\iint_{S_{\epsilon}}\right)\{\cdot\} d S,
$$

where $\{\cdot\}$ represents any function. Evaluating this limit for each term separately yields (the details of the computation are left for Appendix A:

$$
\begin{aligned}
\lim _{\boldsymbol{r}^{\prime} \rightarrow S} \iint_{S} \boldsymbol{A} g d S & =\int_{S} \boldsymbol{A} g d S, \\
\lim _{\boldsymbol{r}^{\prime} \rightarrow S} \iint_{S} \boldsymbol{A} \times \nabla g d S & =\frac{1}{2} \boldsymbol{A} \times \hat{\boldsymbol{n}}+\int \boldsymbol{A} \times \nabla g d S, \\
\lim _{\boldsymbol{r}^{\prime} \rightarrow S} \iint_{S} \nabla \nabla g \cdot \boldsymbol{A} d S & =\oiint_{S} \nabla \nabla g \cdot \boldsymbol{A} d S,
\end{aligned}
$$

where $\boldsymbol{A}=\hat{\boldsymbol{n}} \times \boldsymbol{F}$ and $\boldsymbol{F}$ stands for either the electric or magnetic field. Equations $4 \mathrm{a} 4 \mathrm{~b}$ ) use the Cauchy principal value, denoted by $f f$, and Eq. (4c) the Hadamard finite part [47, Eq. (2.5)], denoted by ff. Substituting the results of Eq. (4) in the limit $\boldsymbol{r}^{\prime} \rightarrow S$ of Eq. (1) yields the hypersingular integral equations

$$
\begin{aligned}
\boldsymbol{E}=\boldsymbol{E}_{\mathrm{inc}} & +\frac{1}{2}(\hat{\boldsymbol{n}} \times \boldsymbol{E}) \times \hat{\boldsymbol{n}} \\
& +\int_{S}\{i k(\hat{\boldsymbol{n}} \times \boldsymbol{B}) g+(\hat{\boldsymbol{n}} \times \boldsymbol{E}) \times \nabla g\} d S \\
& +\oiiint_{S} \frac{i}{k} \nabla \nabla g \cdot(\hat{\boldsymbol{n}} \times \boldsymbol{B}) d S \\
\boldsymbol{B}=\boldsymbol{B}_{\mathrm{inc}} & +\frac{1}{2}(\hat{\boldsymbol{n}} \times \boldsymbol{B}) \times \hat{\boldsymbol{n}} \\
& +\oiiint_{S}\{-i k(\hat{\boldsymbol{n}} \times \boldsymbol{E}) g+(\hat{\boldsymbol{n}} \times \boldsymbol{B}) \times \nabla g\} d S \\
& -\oiiint_{S} \frac{i}{k} \nabla \nabla g \cdot(\hat{\boldsymbol{n}} \times \boldsymbol{E}) d S
\end{aligned}
$$

for $\boldsymbol{r} \in S$. Note that there are no more primed coordinates, as the integral equations (5) are valid only for points on the surface of the mirror. 
Although it may seem surprising for the physical electromagnetic fields to be represented by hypersingular integrals, the singularity is actually caused by the physical discontinuity in the reflecting surface. The hypersingularity in Eqs. (5) disappears if the surface $S$ is closed. Applying Stokes' theorem to the double gradient term in Eqs. (1) yields the usual Stratton-Chu representation for the fields in $V_{\infty}$

$$
\begin{aligned}
& \boldsymbol{E}^{\prime}=\boldsymbol{E}_{\mathrm{inc}}^{\prime}+\frac{1}{i k} \oint_{\partial S} \nabla g \boldsymbol{B} \cdot d \boldsymbol{\ell} \\
& +\int_{S}\{i k(\hat{\boldsymbol{n}} \times \boldsymbol{B}) g+(\hat{\boldsymbol{n}} \times \mathbf{E}) \times \nabla g+(\hat{\boldsymbol{n}} \cdot \boldsymbol{E}) \nabla g\} d S, \\
& \boldsymbol{B}^{\prime}=\boldsymbol{B}_{\mathrm{inc}}^{\prime}-\frac{1}{i k} \oint_{\partial S} \nabla g \boldsymbol{E} \cdot d \boldsymbol{\ell} \\
& +\int_{S}\{i k(\hat{\boldsymbol{n}} \times \boldsymbol{E}) g+(\hat{\boldsymbol{n}} \times \mathbf{B}) \times \nabla g+(\hat{\boldsymbol{n}} \cdot \boldsymbol{B}) \nabla g\} d S .
\end{aligned}
$$

Taking the limit $\boldsymbol{r}^{\prime} \rightarrow S$ as before reveals that all terms diverge at most as $1 / R^{2}$. This divergence can be readily integrated using the Cauchy principal value for the terms inside the surface integral as the integration measure cancels the divergence. However, this is not true for the terms contained in the line integral, as the integration measure, $R$, does not cancel the divergence and the integral is thus hypersingular. It can be shown that, before we take the limit $\boldsymbol{r}^{\prime} \rightarrow S$, the line integral itself vanishes identically for a closed surface [43, thus removing the hypersingularity.

Let us now go back to Eq. (5) and impose the appropriate boundary conditions for a perfectly conducting mirror [48, Eq. (1.18)], i.e.

$$
\hat{\boldsymbol{n}} \times \boldsymbol{E}=0 ; \quad \hat{\boldsymbol{n}} \times \boldsymbol{B}=\boldsymbol{J} .
$$

Extracting the tangential components of Eqs. (5) and imposing these conditions yields

$$
\begin{aligned}
-\hat{\boldsymbol{n}} \times \boldsymbol{E}_{\mathrm{inc}} & =\hat{\boldsymbol{n}} \times\left[\iint_{S} i k \boldsymbol{J} g d S+\oiint_{S} \frac{i}{k} \nabla \nabla g \cdot \boldsymbol{J} d S\right], \\
\frac{1}{2} \boldsymbol{J} & =\boldsymbol{J}_{\mathrm{inc}}+\hat{\boldsymbol{n}} \times \int_{S} \boldsymbol{J} \times \nabla g d S
\end{aligned}
$$

where $\boldsymbol{J}_{\text {inc }}=\hat{\boldsymbol{n}} \times \boldsymbol{B}_{\text {inc }}$. Both equations can be solved for the current $\boldsymbol{J}$ induced by the incident field $\left\{\boldsymbol{E}_{\text {inc }}, \boldsymbol{B}_{\text {inc }}\right\}$. Since it can be shown that the integral operator in the magnetic field integral equation, (8b), is compact, we can use the Liouville-Neumann series to solve the integral equation iteratively [49, §6.16]. The first term of the series yields the usual physical optics approximation (POA)

$$
\boldsymbol{J}=2 \boldsymbol{J}_{\text {inc }},
$$

which coincides with the result for a plane, infinite mirror [50, $\$ 12.2]$. The integral in Eq. 8b can thus be interpreted as a curvature effect. Indeed, each term in the iterative solution can be shown to get gradually smaller in magnitude if the radius of curvature is larger than the wavelength of the incident radiation [50, 51]. The POA has been used successfully in many studies [52, 53].

Substituting the POA [Eq. (9)] and the boundary conditions [Eq. (7)] in Eqs. (1), we can express the reflected field $\boldsymbol{F}_{\text {ref }}^{\prime}=\boldsymbol{F}^{\prime}-\boldsymbol{F}_{\text {inc }}$ in $V_{\infty}$ as

$$
\begin{aligned}
& \boldsymbol{E}_{\mathrm{ref}}^{\prime}=2 \iint_{S}\left\{i k\left(\hat{\boldsymbol{n}} \times \boldsymbol{B}_{\text {inc }}\right) g+\frac{i}{k} \nabla \nabla g \cdot\left(\hat{\boldsymbol{n}} \times \boldsymbol{B}_{\text {inc }}\right)\right\} d S, \\
& \boldsymbol{B}_{\mathrm{ref}}^{\prime}=2 \iint_{S}\left(\hat{\boldsymbol{n}} \times \boldsymbol{B}_{\text {inc }}\right) \times \nabla g d S .
\end{aligned}
$$

Even though Eqs. 10 are valid expressions for the reflected field, the double gradient term tends to strongly oscillate in applications and therefore make its numerical evaluation difficult. We sidestep this issue by once again applying Stokes' theorem on the double gradient term, which leads to

$$
\begin{aligned}
\boldsymbol{E}_{\mathrm{ref}}^{\prime}\left(\boldsymbol{r}^{\prime}, k\right) & =2 \iint_{S}\left\{i k\left(\hat{\boldsymbol{n}} \times \boldsymbol{B}_{\mathrm{inc}}\right) g+\left(\hat{\boldsymbol{n}} \cdot \boldsymbol{E}_{\mathrm{inc}}\right) \nabla g\right\} d S \\
& -\frac{2}{i k} \oint_{\partial S} \nabla g\left[\hat{\boldsymbol{n}} \times\left(\hat{\boldsymbol{n}} \times \boldsymbol{B}_{\mathrm{inc}}\right)\right] \cdot d \boldsymbol{\ell} \\
\boldsymbol{B}_{\mathrm{ref}}^{\prime}\left(\boldsymbol{r}^{\prime}, k\right) & =2 \iint_{S}\left(\hat{\boldsymbol{n}} \times \boldsymbol{B}_{\mathrm{inc}}\right) \times \nabla g d S .
\end{aligned}
$$

The double gradient term has been replaced by two terms: a surface term with a single gradient and an additional line integral term which also contains a single gradient. The single gradient results in a $1 / R$ behavior of the integrands, compared to the $1 / R^{2}$ of the double gradient. This weakens the oscillations of the integrands.

Equations (11) describe a spectral component of frequency $k$ of the reflected field at a given position $\boldsymbol{r}^{\prime}$ as an integral of the incident field on the surface of the mirror. The remainder of this paper will be devoted to their efficient numerical evaluation for arbitrary incident fields $\left\{\boldsymbol{E}_{\text {inc }}, \boldsymbol{B}_{\text {inc }}\right\}$ with complex time-dependence and for arbitrary mirror geometries $S$.

\section{PRACTICAL IMPLEMENTATION}

This section discusses the numerical evaluation of the integrals in Eqs. (11) in some detail. First, we discuss the projection of the incident field on the mirror and introduce the cylindrical coordinate system used throughout the article. Second, we discuss the scale separation that the integral method exhibits, and the domain decomposition strategy that it allows. We also discuss the spatial and temporal discretization schemes used in the numerical implementation. Third, we show that for a specific mirror geometry, the paraboloid mirror, the integrands do not exhibit the strong oscillations typical of diffraction integrals, allowing the use of simple quadrature methods. 


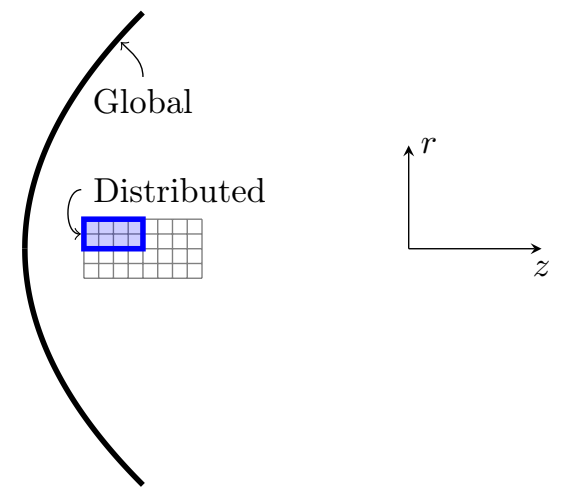

FIG. 2. Domain decomposition method applied to the mesh in the focal region (not to scale). The mesh over the mirror is global, i.e. all processors have a copy of the whole mesh. The mesh in the focal region is distributed over a number of processors. Each processor carries a portion of the whole mesh, and computes the field values for this portion of the mesh only. The blue region depicts the portion that a single processor computes. A cylindrical coordinate system is used.

\section{A. Incident Field Models}

The first step in computing the reflected field in the focal region is to specify the incident field $\left\{\boldsymbol{E}_{\text {inc }}, \boldsymbol{B}_{\text {inc }}\right\}$, i.e. the field that impinges on the mirror $S$. Since the Stratton-Chu formalism does not restrict it in any way, it would in principle be possible to measure the amplitude and phase of each frequency component of the electromagnetic field on the surface of the mirror and use it as an input parameter of Eqs. (11). However, obtaining a complete cartography of the incident field is experimentally very challenging, and it will be more useful to opt for a different approach.

To determine the value of the field on the mirror, we use the Gauss-Laguerre modes of the paraxial wave equation as an expansion basis to describe both linearly and radially polarized beams (see Supplementary Material for explicit expressions). The use of the paraxial approximation is justified in the description of the incident beam as it incurs an error on the order of the divergence angle, i.e. $\mathcal{O}(\epsilon)$. For a highly collimated laser beam, we have $\epsilon \ll 1$. Moreover, since the Stratton-Chu equations are linear, the error in the reflected field will also be $\mathcal{O}(\epsilon)$, even though the reflected field could be tightly focused. The use of these closed-form expressions allows us to easily and quickly evaluate the integrands of Eqs. (11) regardless of the discretization procedure used on the mirror. However, this approach neglects the diffraction of the incident field by the aperture of the mirror. This is justified as the aperture size, $r_{\max }$, is orders of magnitude larger than the wavelength of the incident beam, i.e. $r_{\max } \gg \lambda$ in typical optical experiments.

Since we will consider mostly axisymmetric mirror shapes, such as paraboloid mirrors, we use a cylindrical coordinate system (Fig. 2). The incident field is assumed to be propagating in the $-z$ direction towards the mirror.
For simplicity, and without loss of generality, we assume that the mirror is described by the explicit parametrization $z=F(r, \theta)$. Explicit expressions of Eqs. (11) in these conditions are shown in the Supplementary Material.

\section{B. Temporal Discretization}

In deriving Eqs. 111), we have implicitly assumed that the incident field had been Fourier-transformed, i.e.

$$
\boldsymbol{F}(\boldsymbol{r}, t)=\int_{-\infty}^{\infty} \boldsymbol{F}(\boldsymbol{r}, k) e^{-i k t} d k
$$

where $k=\omega$ is the wavenumber of the radiation. In the numerical implementation the discrete version of the transform is used, i.e.

$$
\boldsymbol{F}(\boldsymbol{r}, t)=\sum_{n=-N_{\max }}^{N_{\max }} \boldsymbol{F}_{n}\left(\boldsymbol{r}, k_{n}\right) e^{-i k_{n} t} \Delta k
$$

where $\boldsymbol{F}_{n}$ is the $n$th frequency sample, or component, $k_{n}$ the sampled frequency and $\Delta k$ the interval between samples. This discrete version is periodic. $N_{\max }$ and $\Delta k$ must be chosen such that the entire duration of the pulse can be represented with Eq. (13) and the Nyquist criterion must be obeyed. To account for the time dependence of the reflected field, the evaluation of the integrals in Eqs. (11) must be performed for each frequency component $\overline{\boldsymbol{F}_{n}}$.

To determine the relative amplitude of each component, we normalize them using a given power spectrum of fixed energy. This is sufficient to uniquely determine the time dependence of the incident beam. Experimentally, it is possible to measure the energy contained in each component $n$. Using the Poynting theorem, we can show that the energy density in each mode is given by the integral of the Poynting vector over an infinite plane, i.e.

$$
\epsilon\left(k_{n}\right)=4 \pi \oiint_{A} \operatorname{Re}\left\{\boldsymbol{E}_{n}\left(\boldsymbol{r}, k_{n}\right) \times \boldsymbol{B}_{n}^{*}\left(\boldsymbol{r}, k_{n}\right)\right\} \cdot d \boldsymbol{A} .
$$

Since Maxwell's equations are linear, each frequency component $\boldsymbol{E}_{n}$ can be renormalized as

$$
\boldsymbol{E}_{n}=E_{0}^{n} \boldsymbol{f}_{E}^{n} ; \quad \boldsymbol{B}_{n}=E_{0}^{n} \boldsymbol{f}_{B}^{n},
$$

where $E_{0}^{n}$ the arbitrary amplitude of the component and $\boldsymbol{f}_{E}\left(\boldsymbol{f}_{B}\right)$ contain the spatial dependence of the field [54]. This allows us to write

$$
E_{0}^{n}=\sqrt{\frac{\epsilon\left(k_{n}\right)}{4 \pi \oiint_{A} \operatorname{Re}\left\{\boldsymbol{f}_{E}\left(\boldsymbol{r}, k_{n}\right) \times \boldsymbol{f}_{B}^{*}\left(\boldsymbol{r}, k_{n}\right)\right\} \cdot d \boldsymbol{A}}}
$$

where $\epsilon\left(k_{n}\right)$ is a known power spectrum, normalized such that

$$
E_{\mathrm{tot}}=\int_{-\infty}^{\infty} \epsilon(k) d k
$$


where $E_{\text {tot }}$ is the total energy of the beam incident on the mirror.

An arbitrary spectral phase relation can be enforced simply by mapping

$$
\begin{aligned}
& \boldsymbol{E}_{\mathrm{inc}}(\boldsymbol{r}, \omega) \mapsto \boldsymbol{E}_{\mathrm{inc}}(\boldsymbol{r}, \omega) e^{i \phi(\omega)}, \\
& \boldsymbol{B}_{\mathrm{inc}}(\boldsymbol{r}, \omega) \mapsto \boldsymbol{B}_{\mathrm{inc}}(\boldsymbol{r}, \omega) e^{i \phi(\omega)},
\end{aligned}
$$

where $\phi(\omega)$ is any function of the frequency. As an example, chirped mirrors can give rise to a frequency dependent phase [26].

To ensure an efficient reconstruction of the time-dependent field, the reflected field should be kept in RAM for the entirety of the computational process. While this implies a larger memory use, it ensures that we do not need to read data from to the disk in the evaluation of the semi-discrete Fourier transform, Eq. (13).

Note that this approach of modeling the time dependence of the field neglects any spatio-temporal couplings [55]. However, the implementation could be updated to support more general time-dependence, such as the position-dependent power spectra that characterizes spatio-temporal couplings.

\section{Spatial Discretization}

The evaluation of Eqs. (11) demands the definition of two separate spatial domains (Fig. 22). The first is the mirror $S$ on which the incident field is assumed known. The second is the region of space in which we wish to compute the reflected field. Since we are mostly interested in the behavior of the reflected field in the vicinity of the focal spot, we will denote this domain the focal region. In the numerical implementation, these two domains are discretized separately. This naturally separates the two length scales that appear in the evaluation of Eqs. (11), as we show in Sec. III C 2.

\section{Meshing the Mirror: Integrand Analysis}

Given the form of Eqs. (11),

$$
I=\int f(\boldsymbol{r}) e^{i k u\left(\boldsymbol{r}, \boldsymbol{r}^{\prime}\right)} d \boldsymbol{r}
$$

where $u\left(\boldsymbol{r}, \boldsymbol{r}^{\prime}\right)=\left|\boldsymbol{r}-\boldsymbol{r}^{\prime}\right|$ is the oscillatory part of the Green's function, one can conclude that the discretization needs to be fine enough to resolve the oscillations of the complex exponential. At first glance, this integrand seems to oscillate rapidly because of the large exponent $k|\boldsymbol{r}| \gg 1$. Indeed, $r$ scales with the size of the parabola $\mathcal{O}(\mathrm{cm})$, while $k \sim \mathcal{O}\left(\mu^{-1}\right)$ for optical pulses. Rapidly oscillating integrands of the form of Eq. 119) are difficult to evaluate numerically, although there are some techniques that facilitate their evaluation 56, 57. Here, however, we show that for a parabolic mirror, a non-trivial cancellation in the phase function results in the strength of the oscillations being controlled by the observation variables $\left(k\left|\boldsymbol{r}^{\prime}\right| \sim 1\right)$ rather than by the integration variables $(k|\boldsymbol{r}| \gg 1)$. It is thus not necessary for the discretization scheme on the mirror to resolve the wavelength, only the variations in the shape of the incident fields $\boldsymbol{f}_{E}$ and $\boldsymbol{f}_{B}$. This allows the use of simple quadrature methods, such as tensor products of either Simpson [58, Eq. (4.1.14)] or Gauss-Legendre [59, §4.6].

The cancellation occurs when we consider the oscillatory behavior of the incoming field. The paraxial fields that are used to represent the incident laser pulses generically have $f(\boldsymbol{r}) \sim e^{-i k z}$ behavior (see Supplementary Material). Hence, the function that needs to be integrated in the exponential above is not $u\left(\boldsymbol{r}-\boldsymbol{r}^{\prime}\right)$, but rather $u\left(\boldsymbol{r}-\boldsymbol{r}^{\prime}\right)-z$. If this function can be shown to be small, i.e. $k u\left(\boldsymbol{r}, \boldsymbol{r}^{\prime}\right)-k z \ll 1$, then the integrands do not have strong oscillations. We examine the phase function in the vicinity of the focal spot, i.e. around $\boldsymbol{r}^{\prime}=0$. We can write the phase function as a bidimensional Taylor series

$$
\begin{aligned}
& u\left(\boldsymbol{r}, \boldsymbol{r}^{\prime}\right)-z=\left(\sqrt{r^{2}+z^{2}}-z\right) \\
& \quad-\frac{r \cos \left(\theta-\theta^{\prime}\right)}{\sqrt{r^{2}+z^{2}}} r^{\prime}-\frac{z}{\sqrt{r^{2}+z^{2}}} z^{\prime}+\mathcal{O}\left(\frac{r^{\prime}, z^{\prime}}{|\boldsymbol{r}|}\right) .
\end{aligned}
$$

Near the focal spot, then, the first-order terms oscillate slowly, because the prefactor of each term has range $[-1,1]$ and $r^{\prime}$ and $z^{\prime}$ (the radial and longitudinal distances from the focal point) do not exceed a few wavelengths, i.e. $k\left|\boldsymbol{r}^{\prime}\right| \sim 1$. The zeroth-order term (parenthesis in Eq. (20) ) is thus the only term that can lead to a rapidly oscillating integrand. For the specific of a parabolic mirror, for which $z=r^{2} / 4 f-f$, the phase function reads

$$
u\left(\boldsymbol{r}, \boldsymbol{r}^{\prime}\right)-z \simeq 2 f-\frac{4 r f}{f^{2}+r^{2}} r^{\prime}-\frac{r^{2}-4 f^{2}}{r^{2}+4 f^{2}} z^{\prime}+\cdots
$$

The zeroth-order term does not depend on the integration variables, implying that the integrand does not oscillate at $\boldsymbol{r}^{\prime}=0$ and oscillates weakly in its vicinity.

This non-trivial cancellation does not carry over to generic surfaces, however. For instance, the zeroth-order term does depend on the integration variables in the ellipsoidal 60] case. In fact, for other mirror geometries, the initial physical motivation of looking at distances not too far away from the focal spot might be lacking. For most mirrors, there are no uniquely defined focal points and the field is diffuse. In these cases, care should be taken when numerically evaluating the Stratton-Chu integrals.

We will use a parabola as the mirror geometry for the remainder of this paper, as it provides the highest field intensities and simplifies the quadrature procedure. 


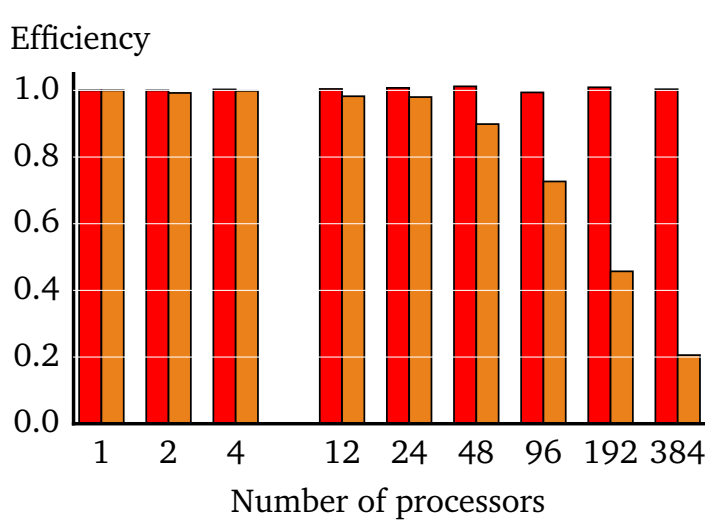

FIG. 3. Parallel efficiency of the algorithm without data output (red) and with data output (orange). If run without data output, the implementation remains very efficient regardless of the number of processors (384 is the maximum that can be requested on the supercomputer on which this test was run). However, the efficiency dramatically drops above 24 processors if there is data output (orange bars). This is due to slow communication between nodes and is not inherent to the algorithm.

\section{Domain Decomposition}

While the mirror does not need to be discretized with sub- $\lambda$ resolution, the focal region requires it. In the tight focusing regime, the focal spot is approximately the size of the incident wavelength, namely $\lambda \sim 1 \mu \mathrm{m}$ for optical lasers, while the mirror and the spatial extension of the incident field usually have centimeter length scales. As hinted on in the introduction, this results in unmanageable memory requirements in traditional computational methods, as they typically need to mesh the entire region of space between the parabola and the focal spot in order to propagate the field between these two regions.

In the Stratton-Chu formalism, only the focal region and the mirror need to be discretized, considerably lowering the memory requirements. However, they can still be prohibitive, requiring a few gigabytes (GBs) per frequency component. This issue is compounded by the fact that temporally short pulses have broad spectra, requiring us to compute Eqs. (11) for a large number of frequency components. Since they must be kept in memory to reconstruct the temporal field, this can lead to data outputs that are approximately 100GB in size, for a typical mesh size of $150 \times 150 \times 150$ points in the focal volume, with $r \in[0,2.5] \mu \mathrm{m}, \theta \in[0,2 \pi]$ and $z \in[-2.5,2.5] \mu \mathrm{m}$. This number may vary significantly as per the incident field model, the number of frequency samples and the strength of the focusing optics.

To mitigate this issue, it is necessary to evaluate Eqs. 111 in parallel. Since the fields at different points in space are computed independently of each other, this can be done efficiently. To achieve maximum efficiency, we use the domain decomposition strategy. Once the fo- cal region has been meshed to the desired accuracy, the mesh is divided onto multiple processors. Each processor contains a portion of the total mesh in its assigned memory, but contains the entirety of the mesh of the mirror (Fig. 2). This way, each processor evaluates the integrals of Eqs. 111 for its portion of the focal spot mesh independently of the others and for each frequency component sequentially. Once the computation is complete, the fields can be written to disk in parallel.

\section{Parallel Efficiency and Runtimes}

We have implemented this algorithm in $\mathrm{C}++$ using the open source OpenMPI parallel library. The output is also parallelized using the parallel version of the HDF5 library. The implementation is efficient (Fig. 3). The efficiency is defined by the metric

$$
E_{n}=\frac{t_{1}}{n t_{n}}
$$

where $n$ is the number of processors and $t_{n}$ is the time it takes to run the program with $n$ processors. Without data output, the algorithm has essentially a perfect parallel efficiency even at a high number of processors $\left(N_{\max }=384\right)$. We surmise, given the embarrassingly parallel nature of the algorithm, that the implementation could scale to a higher number of processors. 384 is the maximum number that can be requested on the supercomputer that is available to our group. The data output, however, is a point of contention. The parallel efficiency drops dramatically when using more than 24 processors (Fig 3). This is caused by the fact that each node (a group of processors that share the same memory) possesses 24 processors. When run on more than 24 processors, the data output function is required to communicate between different nodes that are linked via InfiniBand cables. This bottleneck dramatically increases the runtime of the HDF5 output facilities and thus reduces the parallel efficiency. Note that this is simply a limitation of the cluster's memory architecture, not of our implementation or of the HDF5 library.

In this section, we have discussed the memory requirements and execution times of our algorithm when evaluating the electromagnetic field in a volume around the focal point and for a large number of temporal slices. It is worth putting these numbers into perspective by discussing the cost of evaluating the field at a single point in space, and for a single frequency component, i.e. the cost of a single numerical quadrature over the focusing mirror. For a typical mesh size of $250 \times 250$ points on the reflecting surface, this evaluation takes approximately $0.3 \mathrm{~s}$ on a single core of an Intel Core i7-4700MQ CPU. The computation time scales linearly with the number of points used on the mesh of the mirror and also with the number of frequency components. If one needs to compute the field at a given point in time, all field components should be computed at the same spatial point and the 
semi-discrete Fourier transform should be taken. Usually, the Fourier transform takes a negligible amount of time compared to the field calculation. For a typical optical 20 fs pulse, 50 frequency components suffice to accurately sample the power spectrum and computing the field at a single space-time point takes $50 \times 0.3 \mathrm{~s}=15 \mathrm{~s}$.

\section{FIELDS IN THE FOCAL REGION}

The unavailability of closed-form solutions of Eq. (11) makes a systematic verification of any numerical implementation of the Stratton-Chu diffraction integrals difficult. However, since they represent physical fields, the values calculated with our implementation should obey Maxwell's equations and should satisfy the principle of energy conservation. We verify that the reflected fields computed via our implementation converge as we reduce the discretization size, and that they obey the previously listed conditions.

\section{A. Numerical Checks}

In this section, we study the convergence of the evaluation of the integrals in Eqs. (11). We also verify that the computed fields obey Maxwell's equations in both the frequency and time domains and that our implementation conserves the energy of the system.

We use a radially polarized Gaussian beam as the incident field (see Supplementary Material for the explicit expressions). We also assume that its spectral power is super-Gaussian in $\lambda$ and that the full-width halfmaximum (FWHM) temporal duration associated with the spectrum is $18 \mathrm{fs}$ (see Table I for detailed parameters). The parabolic mirror has a numerical aperture of $\mathrm{NA}=1$, i.e. $r_{\max }=2 f$. The transverse width of the incident field is chosen such that the field is not clipped by the edge of the mirror. While we present our test results only for the radial polarization to save on space, we have verified that the results hold also for linearly polarized fields.

To verify that our results have converged, we evaluate the reflected field, i.e. the surface integrals in Eqs. (11), for different mesh sizes. We then use the results obtained with the finest mesh as a reference to measure the convergence speed. To do so, we compute the relative error, defined as the maximum value of the absolute difference of the fields over the focal region divided by the maximum value of the magnitude of the reference field

$$
e_{\text {rel }}=\frac{\max _{\boldsymbol{r}^{\prime}}\left|\boldsymbol{F}_{N_{\max }}-\boldsymbol{F}_{N}\right|}{\max _{\boldsymbol{r}^{\prime}}\left|\boldsymbol{F}_{N_{\max }}\right|}
$$

where $\boldsymbol{F}_{N}$ is the electromagnetic field computed with $N$ radial discretization points. Because we use cylindrical coordinates, the cells of the mesh increase in area with

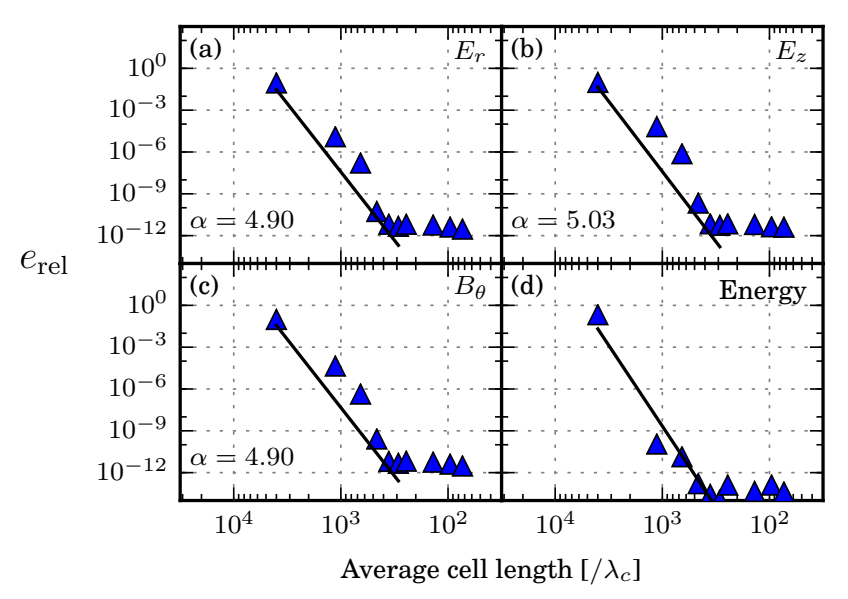

FIG. 4. (a)-(c) Relative error $e_{\text {rel }}$ of each component of the radially polarized electromagnetic field in the focal spot (blue triangles) as a function of the average polar cell length given as a fraction of the central wavelength $\lambda_{c}$. The slope $\alpha$ of the linear fit (black curves) shows that the convergence order is given by the order of the quadrature method for each component. (d) Relative difference between the energy of the incident beam and the energy of the field in the focal spot. The linear fit (black curve) shows a convergence order consistent with the energy being a quadratic function of the fields. See Table I for the simulation parameters used to generate this figure.

the distance from the origin. We use the average area,

$$
\langle A\rangle_{N}=\frac{\Delta \theta(\Delta r)^{2}}{2} \sum_{n=0}^{N}(2 n+1)=\frac{\Delta \theta(\Delta r)^{2}}{2} \frac{(N+1)^{2}}{N},
$$

where $\Delta \theta$ is the angular mesh resolution, $\Delta r$ the radial mesh resolution and $N$ of radial mesh points, as a measure of the mesh resolution. Each individual component converges according to the order of the quadrature method that was used to evaluate the integrands. Specifically, we used a fifth order method, and the components converge following $h^{-\alpha}$ with $\alpha \sim 5$ (Fig. 4 a-c).

It is interesting to note that the typical mesh size necessary for convergence (the saturated portions of Fig. 4) is more than a hundred (100) times larger than the wavelength of the beam. This is a direct consequence of the results of Sec. IIIC1.

Our implementation also satisfies the principle of conservation of energy and its numerical value converges following the order of the quadrature method, same as the individual components (Fig. 4 $\mathrm{d}$ ). To verify this, we have computed the total energy in the domain given by (in SI units)

$$
E=\frac{1}{2} \iiint_{V}\left[\epsilon_{0} \boldsymbol{E}(\boldsymbol{r}, t)^{2}+\frac{1}{\mu_{0}} \boldsymbol{B}(\boldsymbol{r}, t)^{2}\right] d V
$$

as a function of the average mesh size. This quantity is equal to the integral of the energy density in the frequency domain, Eq. (17). 
To ensure that the electromagnetic energy contained in the focal region and the energy of the incident beam are numerically equal, we must make sure that the spatial extension of the incident beam is smaller than the aperture of the mirror so it is not clipped. It is also necessary to compute the reflected field in a region of space that spans several wavelengths, on the order of $25 \lambda$ in each direction. This is substantially larger than the $\lambda^{3}$ volume we are usually interested in. This is because the longitudinal components have a larger spatial extension than the transverse ones (see next section for a discussion). In turn, the larger focal region forces us to use a finer mesh on the parabola, as the integrands oscillate more strongly at larger distances from the geometrical focal point, as discussed in Sec. III.

The fields in the focal spot have numerically been shown to obey Maxwell's equations, in both the frequency and time domains (Fig. 5). To show this, we computed the magnitude of the relative difference vectors

$$
\begin{aligned}
\boldsymbol{e}_{\mathrm{f}}=\frac{2(\nabla \times \boldsymbol{E}-i k \boldsymbol{B})}{\max _{\boldsymbol{r}^{\prime}}|\nabla \times \boldsymbol{E}|+\max _{\boldsymbol{r}^{\prime}}|i k \boldsymbol{B}|} \\
+\frac{2(\nabla \times \boldsymbol{B}+i k \boldsymbol{E})}{\max _{\boldsymbol{r}^{\prime}}|\nabla \times \boldsymbol{B}|+\max _{\boldsymbol{r}^{\prime}}|i k \boldsymbol{E}|}, \\
\boldsymbol{e}_{\mathrm{t}}=\frac{2\left(\nabla \times \boldsymbol{E}+\partial_{t} \boldsymbol{B}\right)}{\max _{\boldsymbol{r}^{\prime}}|\nabla \times \boldsymbol{E}|+\max _{\boldsymbol{r}^{\prime}}\left|\partial_{t} \boldsymbol{B}\right|} \\
+\frac{2\left(\nabla \times \boldsymbol{B}-\partial_{t} \boldsymbol{E}\right)}{\max _{\boldsymbol{r}^{\prime}}|\nabla \times \boldsymbol{B}|+\max _{\boldsymbol{r}^{\prime}}\left|\partial_{t} \boldsymbol{E}\right|},
\end{aligned}
$$

which should vanish if the fields obey Maxwell's equations. The curls and time derivative are computed via a central finite difference scheme on the mesh. This specific definition for the relative difference is chosen as to provide a natural scale for the quantities in the numerator of Eq. 26. .

Equation (26a) tests the Stratton-Chu quadrature routines that evaluate Eqs. (11), as we perform all in our calculations in the frequency domain. Equation $26 \mathrm{~b}$ tests the temporal reconstruction routine, i.e. our implementation of Eq. (13).

\section{B. Tightly Focused Linearly Polarized Gaussian Beam}

In this section, we show a concrete example of a calculation that can be performed using our efficient implementation of the Stratton-Chu integral representation. We study the spatio-temporal focusing of a linearly polarized (along the $x$-axis), paraxial femtosecond pulse incident onto a high numerical aperture on-axis parabola. A similar setup was first used in a scheme to directly accelerate electrons with a high-intensity radially polarized field 61 64.

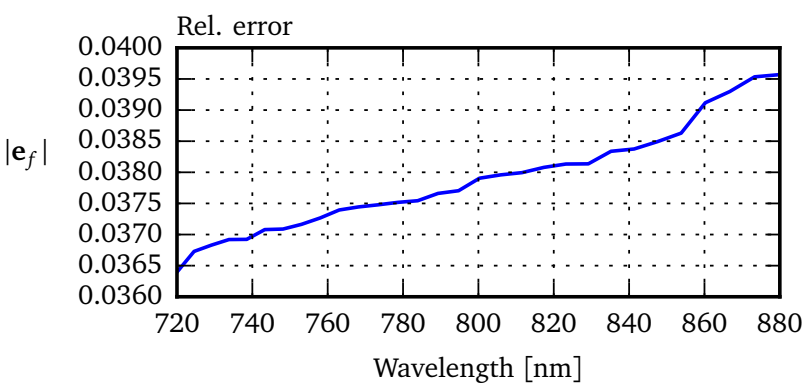

\begin{tabular}{|c|c|c|c|c|c|}
\hline \multicolumn{6}{|c|}{ Simulation Parameters } \\
\hline \multicolumn{3}{|c|}{ Parabola } & \multicolumn{3}{|c|}{ Incident Beam } \\
\hline Param. & Value & Unit & Param. & Value & Unit \\
\hline$r_{\max }$ & 0.125 & $\mathrm{~m}$ & $w_{0}$ & 0.100 & $\mathrm{~m}$ \\
\hline \multirow[t]{4}{*}{$f$} & 0.0675 & $\mathrm{~m}$ & $\lambda_{c}$ & 820 & $\mathrm{~nm}$ \\
\hline & & & $\Delta \lambda$ & 70 & $\mathrm{~nm}$ \\
\hline & & & $n$ & 3 & - \\
\hline & & & $E_{\text {tot }}$ & 150 & $\mathrm{~J}$ \\
\hline
\end{tabular}

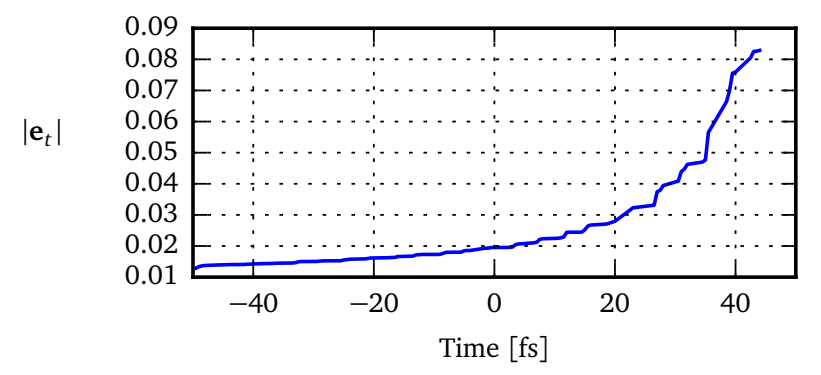

FIG. 5. Average value of the magnitude of the relative error vectors $\boldsymbol{e}_{f}$ and $\boldsymbol{e}_{t}$ over the focal region.

TABLE I. Simulation parameters used in the numerical calculations. They were chosen as to best match the specifications of planned high-power laser facilities [1, 65].

The relevant parameters of the simulation are defined in Fig. 6. The shape of the parabola is fixed by its focal length $f$ and its aperture size $r_{\max }$. The numerical aperture is determined geometrically from the opening angle of the parabola and is given by $\mathrm{NA}=\sin \theta$. In Fig. 6(a), the parabola has NA $=1$. The power spectrum, shown in Fig. 6(b), is chosen to have a super-Gaussian shape

$$
\epsilon(\lambda) \propto \exp \left[\left(\frac{\lambda-\lambda_{c}}{\Delta \lambda}\right)^{2 n}\right],
$$

which corresponds to the time dependence pictured in Fig. 6(c). The numerical values of the parameters were chosen as to best approximate the specifications of planned high-power laser facilities [1, 65, 66] and are shown in Table [1]

The structure of the field in the geometric focal plane (Fig. 7) exhibits features that cannot be replicated within the paraxial approximation, nor even by using vacuum solutions of Maxwell's equations, e.g. [27, 67. The $E_{x}$ 

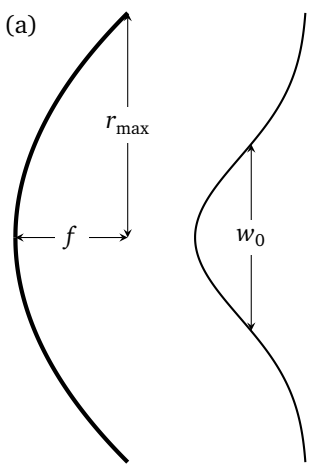

(b)

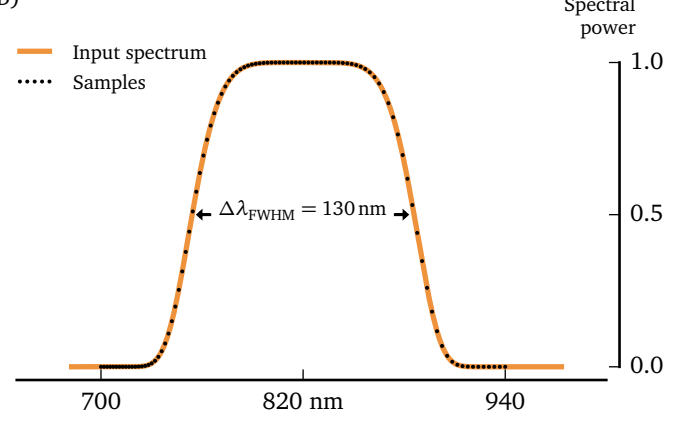

(c)

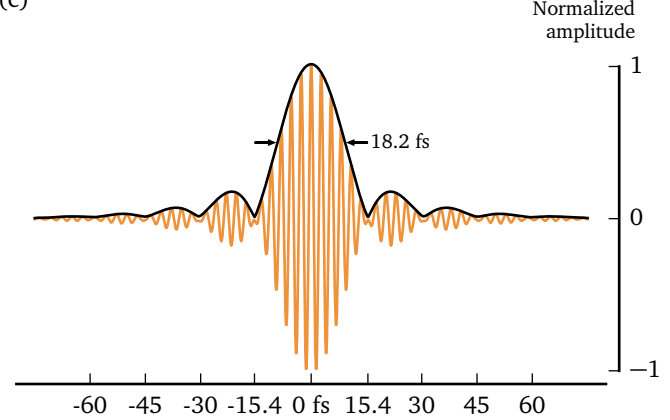

FIG. 6. Characteristics of the incident beam. (a) A Gaussian beam with a beam waist $w_{0}$ impinges on parabolic mirror of focal length $f$ and aperture size $r_{\max }$. (b) The beam has a super-Gaussian power spectrum centered at $\lambda_{c}=820 \mathrm{~nm}$ and has a full width at half maximum (FWHM) of $130 \mathrm{~nm}$. We used 100 frequency samples (black dots) in the numerical simulation. (c) In the time domain, this corresponds to a field that has a main pulse with FWHM duration of $18.2 \mathrm{fs}$ and weaker, shorter revivals at earlier and later times.

and $B_{y}$ components, the only non-vanishing components of the incident beam, dominate the irradiance distribution. The reflection imparts upon them an elliptical structure with the major axis in the $x$ direction, i.e. parallel to the laser polarization. This ellipticity disappears at low enough numerical apertures, i.e. in the paraxial regime. Moreover, the fields acquire relatively strong longitudinal components, with the magnitude of $B_{z}$ being as large as half the $E_{x}$ component 41 . These relatively large longitudinal field components are conspicuously absent from low numerical aperture simulation results, confirming that they originate from the strong curvature of the parabolic mirror. Indeed, comparing transverse cuts of the longitudinal field $E_{z}$ and $B_{z}$ in the focal plane for different numerical apertures $(\mathrm{NA}=1$ and $\mathrm{NA}=0.7$ ) shows that the longitudinal fields decrease with lower numerical aperture (Fig. 8). These results highlight the importance of modeling techniques which account for the reflection off high numerical aperture mirrors, such as the Stratton-Chu diffraction integrals.

This more accurate beam model could have repercussions in the modeling of physical processes in the presence of high-intensity laser beams. For instance, the presence of strong longitudinal components with a smoother beam profile than the transverse components will affect the trajectories of charged particles interacting with the laser in the focal spot, due to the different ponderomotive forces in each direction. Furthermore, the resulting field invariants, which are qualitatively different than those that occur at low numerical apertures, can also result in the enhancement of the pair production and vacuum polarization signatures 4 .

The maximum electrical intensity, defined by (in SI units)

$$
I_{E}=\frac{1}{2} c \epsilon_{0} \boldsymbol{E}^{2}
$$

is of interest for SF-QED applications, as most observables strongly depend on this parameter. It can be shown to scale linearly with the total energy of the incident beam (via 16 ). For fixed beam waist, simulations show that the intensity increases quadratically with increasing numerical aperture (not shown).

In particular, our simulations show that, electrical intensities of up to $5 \times 10^{24} \mathrm{~W} / \mathrm{cm}^{2}$ could be obtained (Fig. 9). The electrical intensity has a discernible elliptical structure. It is caused both by the ellipticity in the $E_{x}$ component and, to a smaller extent, by the off-center shape of the $E_{z}$ component. At these intensities, it may be feasible to experimentally detect radiation reaction 68] and vacuum four-wave mixing [7].

Note that this intensity value does not take into account the possibilitiy of an imperfect vacuum or of Schwinger pair creation, both of which could trigger a QED cascade and deplete the laser energy [69, 70].

\section{CONCLUSION}

We used the Stratton-Chu integral representation to model the reflection of temporally short laser beams off strongly focusing optics. We have shown that while the integral representation leads to hypersingular integral equations for the reflected fields in the case of an open surface, the resulting magnetic field integral equation still has a compact operator and can be solved iteratively with the Liouville-Neumann series. We have shown that this approach yields the physical optics approximation. We then generalized this monochromatic technique to handle temporally short pulses, or, in other words, polychromatic fields. The formalism is of experimental interest, as its input is the unfocused laser field coming from the laser system. Usually, this field is experimentally characterized with a high level of accuracy, and this measurement could in principle be used as input data for the Stratton-Chu formalism.

We then discussed the development of an efficient parallel, numerical evaluation of the integrals. It is shown 

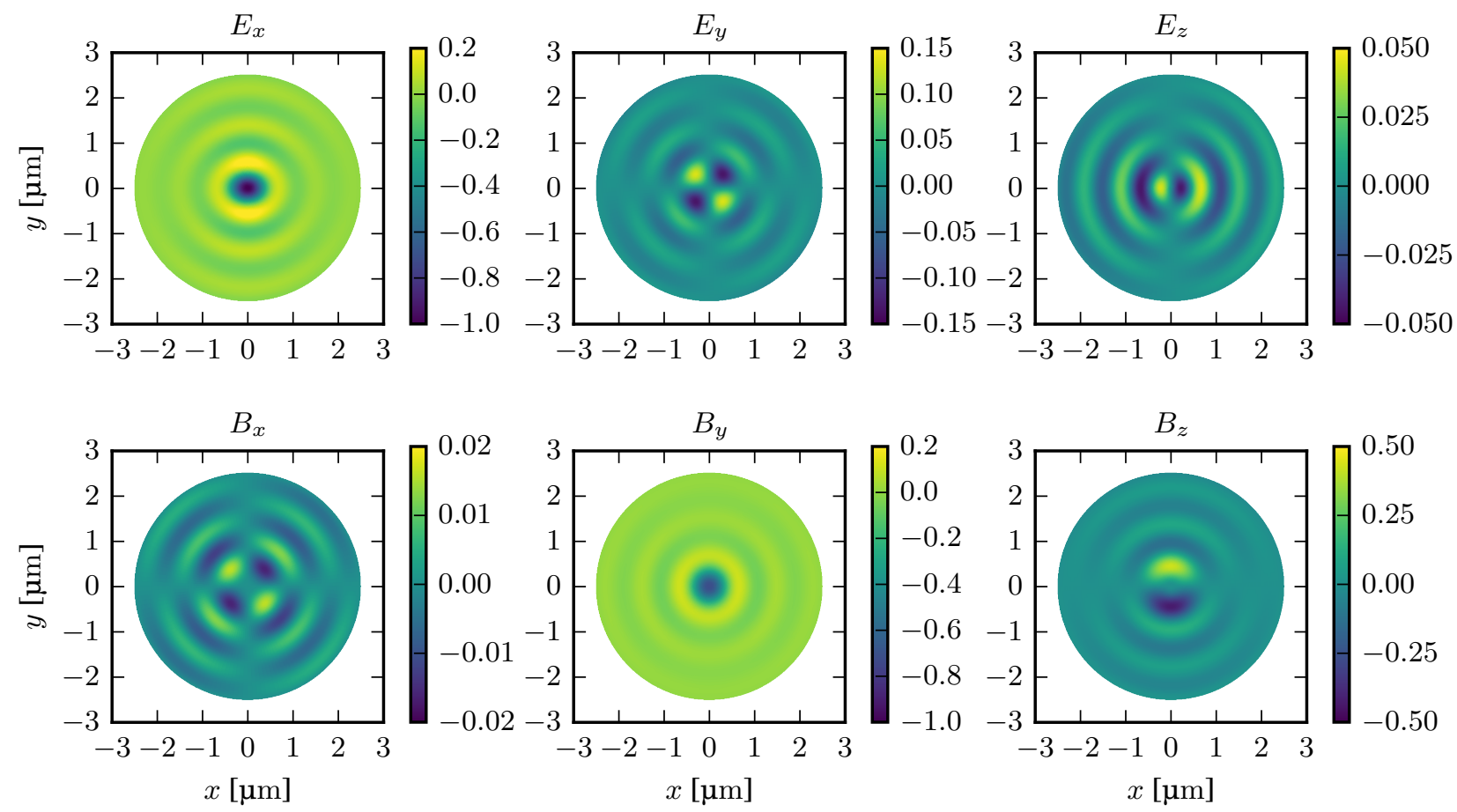

FIG. 7. Components of time-dependent electromagnetic field in the focal plane of the parabolic mirror at the time at which $I_{E}$ is maximum. The components are scaled with respect to the maximum field magnitude across all components. The incident beam is polarized in the $x$ direction (horizontal axis in the figure). The simulation parameters used to obtain these results are shown in Table I

that, unexpectedly, the integrands do not strongly oscillate in the specific case of a parabolic mirror. This allows the use of simple quadrature methods (e.g. GaussLegendre), instead of specialized quadrature methods. We have shown that the integral method naturally separates the mirror and focal meshes. As a consequence, the memory requirements are dramatically lessened as compared to traditional FDTD, FDFD and FEM methods. This separation also allowed for a highly efficient parallel implementation of the algorithm. We have shown that our implementation can be scaled to at least 384 processors.

In the last section, we verified that our implementation converges properly as a function of the mesh size and that the resulting fields are solutions of Maxwell's equations in both the frequency and time domain. We also verified that the algorithm conserves the energy of the incident beam.

We showed that future laser facilities, such as ELI and APOLLON, could obtain focused intensities on the order of $10^{24} \mathrm{~W} / \mathrm{cm}^{2}$. The dominant components of the linearly polarized incident beam, $E_{x}$ and $B_{y}$, acquire an elliptical structure upon reflection from a parabolic mirror, with the major axis being parallel to the polarization. Longitudinal components as large as $0.5 E_{x}$ also appear in the reflected field. Both of these effects are due to the strong curvature of the high numerical aperture parabolic mirror.

\section{ACKNOWLEDGEMENTS}

The authors thank S. Payeur, S. Fourmaux, A. Lachapelle and J.-C. Kieffer for helpful discussions. J.D. gratefully acknowledges financial support from FRQNT. Computations were made on the supercomputer MP2 from Université de Sherbrooke, managed by Calcul Québec and Compute Canada. The operation of this supercomputer is funded by the Canada Foundation for Innovation (CFI), the ministère de l'Économie, de la science et de l'innovation du Québec (MESI) and the Fonds de recherche du Québec - Nature et technologies (FRQNT). We wish to thank H. Z. Lu for his tremendous technical support. We also acknowledge the software packages matplotlib, used to create some of the figures [71, and GNU parallel [72, used in the data analysis.

\section{Appendix A: Evaluation of the surface integrals at the singular point}

Here, we evaluate the singular part of the integrals of the Stratton-Chu equations, Eqs. (4). The integration is done over a hemispherical surface of radius $R$ centered at $\boldsymbol{r}=\boldsymbol{r}^{\prime}$. We use spherical coordinates $(R, \phi, \theta)$. Note that $\boldsymbol{R}=\boldsymbol{r}-\boldsymbol{r}^{\prime}$ in these coordinates. We then take the limit as $R \rightarrow 0$. Since the surface is small, we suppose that the field does not change on the surface and assume it 

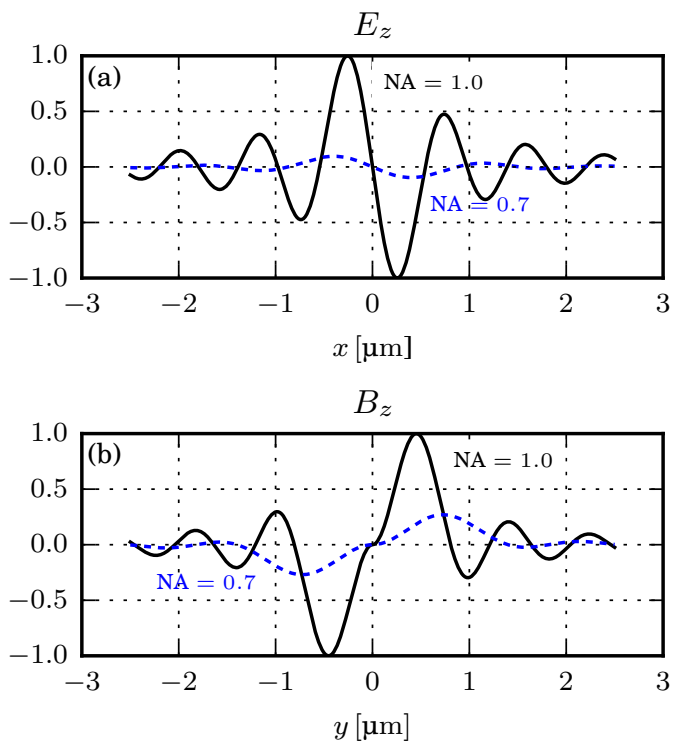

FIG. 8. Cuts of the longitudinal components of the electromagnetic field in the focal plane for parabolic mirrors of numerical aperture $\mathrm{NA}=1$ and $\mathrm{NA}=0.7$. The amplitudes are normalized with respect to the maximum value of the electric and magnetic field in the NA $=1$ case. (a) The cut of the electric field is along the $x$ direction. (b) The cut of the magnetic field is along the $y$ direction. Both electric and magnetic longitudinal field components decrease quadratically with numerical aperture (not shown).

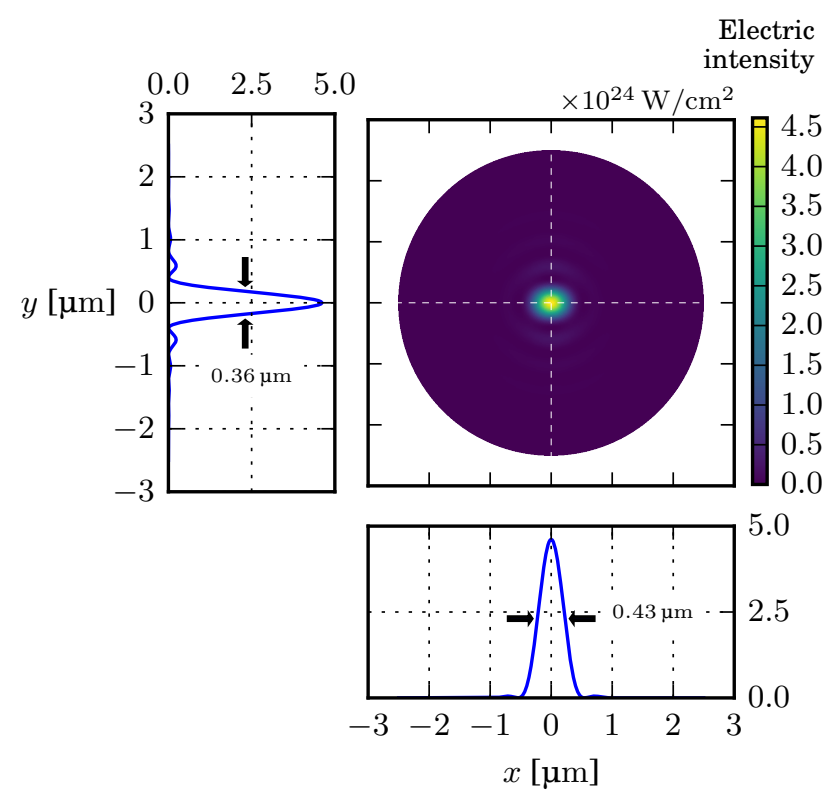

FIG. 9. Electric intensity in the focal plane in $\mathrm{W} / \mathrm{cm}^{2}$ and transverse cuts through the focal point (dashed lines). The ellipticity manifests itself in different full width at halfmaximum in the transverse cuts. The resulting eccentricity is approximately $e \simeq 0.55$. takes its value at $\boldsymbol{r}=\boldsymbol{r}^{\prime}$ over the whole surface. We can then move it outside the integral sign. We also ignore the phase of the Green's function, as it is constant over the hemisphere and goes to 0 after the limit. The integral over the hemisphere in Eq. 4a becomes

$$
\begin{aligned}
\lim _{R \rightarrow 0} \iint_{S_{\epsilon}} \boldsymbol{A} g d S & =\lim _{R \rightarrow 0} \boldsymbol{A} \int_{0}^{\frac{\pi}{2}} \int_{0}^{2 \pi} \frac{1}{4 \pi R} R^{2} \sin \theta d \phi d \theta \\
& =0
\end{aligned}
$$

The integral is in fact regular at $\boldsymbol{r}=\boldsymbol{r}^{\prime}$ because the integration measure cancels the pole of the Green's function.

In Eq. (4b), the gradient of the Green's function generates a $R^{-1}$ term and a $R^{-2}$ term. The former does not contribute to the integral because of the integration measure, while the latter reads

$$
\begin{aligned}
\lim _{R \rightarrow 0} \iint_{S_{\epsilon}} \boldsymbol{A} \times \nabla g d S= & \lim _{R \rightarrow 0} \boldsymbol{A} \times \\
& \int_{0}^{\frac{\pi}{2}} \int_{0}^{2 \pi} \frac{R^{2} \hat{\boldsymbol{n}}}{4 \pi R^{2}} \sin \theta d \phi d \theta, \\
& =\frac{1}{2} \boldsymbol{A} \times \boldsymbol{n},
\end{aligned}
$$

where $\boldsymbol{n}$ comes from the fact that the gradient of the Green's function is normal to the hemispherical surface.

The integral in Eq. 4c diverges due to the presence of the double gradient of $g$. However, it is still possible to assign it a finite value. To do so, we use the explicit expression of the double gradient of the Green's function 73. Eq. (2.61)]:

$$
\begin{aligned}
\nabla \nabla g\left(\boldsymbol{r}, \boldsymbol{r}^{\prime}\right) & =-\overline{\overline{\mathbf{I}}}\left[-\frac{i k}{R}+\frac{1}{R^{2}}\right] g \\
& +(\hat{\boldsymbol{R}} \otimes \hat{\boldsymbol{R}})\left[\frac{3}{R^{2}}-\frac{3 i k}{R}-k^{2}\right] g
\end{aligned}
$$

where $\overline{\overline{\mathbf{I}}}$ is the unit dyad, $R=\left|\boldsymbol{r}-\boldsymbol{r}^{\prime}\right|, \hat{\boldsymbol{R}}=\left(\hat{\boldsymbol{r}}-\hat{\boldsymbol{r}}^{\prime}\right) / R$ and $\otimes$ is the Kronecker outer product. The contribution of the second line of Eq. A3 to the surface integral vanishes due to the tensor structure, i.e.

$$
\hat{\boldsymbol{R}} \otimes \hat{\boldsymbol{R}} \cdot(\hat{\boldsymbol{n}} \times \boldsymbol{F}) \stackrel{r \in S_{\epsilon}}{\longrightarrow} \hat{\boldsymbol{R}} \otimes \hat{\boldsymbol{R}} \cdot(\hat{\boldsymbol{R}} \times \boldsymbol{F}) .
$$

On the hemispherical surface, the dyad $\hat{\boldsymbol{R}} \otimes \hat{\boldsymbol{R}}$ has a single non-vanishing component in, obviously, the $\hat{\boldsymbol{R}} \otimes \hat{\boldsymbol{R}}$ direction, while the vector it multiplies, $\hat{\boldsymbol{R}} \times \boldsymbol{F}$ only has angular $(\hat{\boldsymbol{\phi}}$ and $\hat{\boldsymbol{\theta}})$ components. The dyad-vector product of Eq. A4 thus vanishes. The contribution of the first line of Eq. (A3), however, does not vanish. We expand it in a Laurent-type series and obtain

$$
\begin{aligned}
\left(-\frac{i k}{R}+\frac{1}{R^{2}}\right) g & \simeq\left(-\frac{i k}{R^{2}}+\frac{1}{R^{3}}\right)(1+i k R) \\
& =-\frac{i k}{R^{2}}+\frac{1}{R^{3}}+\frac{k^{2}}{R}+\frac{i k}{R^{2}} \\
& =\frac{1}{R^{3}}+\frac{k^{2}}{R}
\end{aligned}
$$


Substituting this last expression in the l.h.s of Eq. 4c, we see that the second term vanishes due to the $R^{2}$ measure and that the first term formally diverges. To ascribe a finite value to this integral, we make use of the Hadamard finite part, which essentially drops the diverg- ing term in a mathematically consistent way [47. Equation (4c) thus reads

$$
\lim _{\boldsymbol{r}^{\prime} \in S} \iint_{S} \nabla \nabla g \cdot(\hat{\boldsymbol{n}} \times \boldsymbol{F}) d S=\oiiint_{S} \nabla \nabla g \cdot(\hat{\boldsymbol{n}} \times \boldsymbol{F}) d S .
$$

[1] D. Papadopoulos, C. L. Blanc, G. Chériaux, P. Georges, G. Mennerat, J. P. Zou, F. Mathieu, and P. Audebert, in Advanced Solid-State Lasers Congress (Optical Society of America, 2013) p. ATu3A.43.

[2] C. Danson, D. Hillier, N. Hopps, and D. Neely, High Power Laser Sci. Eng. 3, e3 (2015)

[3] A. M. Fedotov, Laser Phys. 19, 214 (2009)

[4] Y. Monden and R. Kodama, Phys. Rev. Lett. 107, $073602(2011)$

[5] A. Di Piazza, C. Müller, K. Z. Hatsagortsyan, and C. H. Keitel, Rev. Mod. Phys. 84, 1177 (2012)

[6] A. Titov, B. Kämpfer, H. Takabe, and A. Hosaka, Phys. Rev. A 87, 042106 (2013).

[7] F. Fillion-Gourdeau, C. Lefebvre, and S. MacLean, Phys. Rev. A 91, 031801(R) (2015)

[8] G. A. Mourou, T. Tajima, and S. V. Bulanov, Rev. Mod. Phys. 78, 309 (2006)

[9] G. Mourou, J. A. Wheeler, and T. Tajima, Europhys. News 46, 31 (2015).

[10] M. Lax, W. H. Louisell, and W. B. McKnight, Phys. Rev. A 11, 1365 (1975)

[11] Y. I. Salamin, New J. Phys. 8, 133 (2006)

[12] W. v. Ignatowsky, Ann. Phys. 23, 875 (1907).

[13] W. v. Ignatowsky, Ann. Phys. 25, 99 (1908).

[14] F. Kottler, Ann. Phys. 70, 405 (1923).

[15] F. Kottler, Ann. Phys. 71, 457 (1923).

[16] J. Stratton and L. Chu, Phys. Rev. 56, 99 (1939).

[17] E. Wolf, Proc. R. Soc. A Math. Phys. Eng. Sci. 253, 349 (1959).

[18] B. Richards and E. Wolf, Proc. R. Soc. A Math. Phys. Eng. Sci. 253, 358 (1959)

[19] P. Varga and P. Török, J. Opt. Soc. Am. A 17, 2081 (2000)

[20] M. Lieb and A. Meixner, Opt. Express 8, 458 (2001).

[21] S. Quabis, R. Dorn, M. Eberler, O. Glöckl, and G. Leuchs, Appl. Phys. B 72, 109 (2001)

[22] S.-W. Bahk, P. Rousseau, T. A. Planchon, V. Chvykov, G. Kalintchenko, A. Maksimchuk, G. A. Mourou, and V. Yanovsky, Appl. Phys. B 80, 823 (2005).

[23] N. Bokor and N. Davidson, Opt. Commun. 281, 5499 (2008)

[24] A. April, Opt. Lett. 33, 1563 (2008)

[25] A. April and M. Piché, Opt. Express 18, 22128 (2010).

[26] T. M. Jeong, S. Weber, B. Le Garrec, D. Margarone, T. Mocek, and G. Korn, Opt. Express 23, 11641 (2015)

[27] C. J. R. Sheppard and S. Saghafi, J. Opt. Soc. Am. A 16, 1381 (1999)

[28] I. Gonoskov, A. Aiello, S. Heugel, and G. Leuchs, Phys. Rev. A 86, 053836 (2012).

[29] Y. I. Salamin, Phys. Rev. A 92, 053836 (2015).

[30] J.-P. Berenger, J. Comput. Phys. 114, 185 (1994).

[31] F. Hebenstreit, Schwinger effect in inhomogeneous electric fields, Ph. D. thesis, Karl-Franzens-Universität Graz
(2011), arXiv:1106.5965

[32] C. Schneider and R. Schützhold, J. High Energy Phys. 2016, 1 (2016).

[33] H. Gies, F. Karbstein, and N. Seegert, Phys. Rev. D 93, 085034 (2016), arXiv:1603.00314

[34] F. Mackenroth and A. Di Piazza, Phys. Rev. A 83, 032106 (2011)

[35] A. Di Piazza, Phys. Rev. Lett. 113, 040402 (2014).

[36] A. Di Piazza, Phys. Rev. A 91, 042118 (2015).

[37] A. Di Piazza, Phys. Rev. Lett. 117, 213201 (2016)

[38] Y. I. Salamin, Opt. Lett. 32, 90 (2007)

[39] C. Varin, S. Payeur, V. Marceau, S. Fourmaux, A. April, B. Schmidt, P.-L. Fortin, N. Thiré, T. Brabec, F. Légaré, J.-C. Kieffer, and M. Piché, Appl. Sci. 3, 70 (2013).

[40] V. Marceau, C. Varin, and M. Piché, Opt. Lett. 38, 821 (2013)

[41] K. Youngworth and T. Brown, Opt. Express 7, 77 (2000)

[42] V. Marceau, C. Varin, T. Brabec, and M. Piché, Phys. Rev. Lett. 111, 224801 (2013)

[43] M. I. Sancer, Radio Sci. 3, 141 (1968).

[44] A. J. Poggio and E. Miller, in Computer Techniques for Electromagnetics, International Series of Monographs in Electrical Engineering, edited by R. Mittra (Pergamon, 1973) pp. $159-264$.

[45] E. L. Roetman, R. P. Kochhar, and G. L. Hower, Electromagnetics 12, 1 (1992).

[46] C.-T. Tai, Generalized Vector and Dyadic Analysis, 2nd ed., IEEE/OUP Series on Electromagnetic Wave Theory (Wiley-Interscience, 1997).

[47] L. Blanchet and G. Faye, J. Math. Phys. 41, 7675 (2000)

[48] J. A. Stratton, Electromagnetic Theory (McGraw-Hill, 1941).

[49] D. S. Jones, Methods in Electromagnetic Wave Propagation, 2nd ed., IEEE/OUP Series on Electromagnetic Wave Theory (Wiley-IEEE Press, 1994).

[50] J. van Bladel, Electromagnetic Fields, 2nd ed., IEEE Press Series on Electromagnetic Wave Theory (WileyInterscience, 2007).

[51] J. A. Cullen, Phys. Rev. 109, 1863 (1958)

[52] C. J. Bouwkamp, Rep. Prog. Phys. 17, 302 (1954)

[53] A. W. Love, ed., Reflector Antennas, IEEE Press Selected Reprint Series (IEEE Press, 1978).

[54] While these functions are arbitrary, we evaluate this integral for specific beam models in the Supplementary Material.

[55] G. Pariente, V. Gallet, A. Borot, O. Gobert, and F. Quéré, Nat. Photonics 10, 547 (2016).

[56] A. Iserles and S. P. Nørsett, BIT Numer. Math. 44, 755 (2004)

[57] M. Ganesh, S. Langdon, and I. Sloan, J. Comput. Appl. Math. 204, 363 (2007).

[58] W. H. Press, Numerical Recipes in Pascal (First Edition): The Art of Scientific Computing, Volume 1 (Cambridge 
University Press, 1989).

[59] W. H. Press, S. A. Teukolsky, W. T. Vetterling, and B. P. Flannery, Numerical Recipes : the Art of Scientific Computing, 3rd ed. (Cambridge University Press, 2007).

[60] More precisely, mirrors of the form $z^{2} / c^{2}=1-\left(x^{2}+\right.$ $\left.y^{2}\right) / a^{2}$, with focal spots at $z= \pm \sqrt{c^{2}-a^{2}}$.

[61] S. Payeur, S. Fourmaux, B. E. Schmidt, J. P. MacLean, C. Tchervenkov, F. Légaré, M. Piché, and J. C. Kieffer, Appl. Phys. Lett. 101, 041105 (2012)

[62] J. P. MacLean, S. Payeur, S. Fourmaux, C. Tchervenkov, B. Schmidt, P. Lassonde, F. Légaré, M. Piché, and J. C. Kieffer, in Proc. SPIE: Penetrating Radiation Systems and Applications XIII, Vol. 8509 (2012) p. 85090K.

[63] S. Payeur, S. Fourmaux, J. Kieffer, M. Piché, J. P. MacLean, and C. Tchervenkov, "All-optical method and system for generating ultrashort charged particle beam," (2013), US Patent 8,558,199.

[64] A. Lachapelle, K. Otani, S. Fourmaux, S. Payeur, M. Glesser, S. MacLean, and J. C. Kieffer, in Proc. SPIE 9515, Research Using Extreme Light: Entering New Frontiers with Petawatt-Class Lasers II , edited by G. Korn and L. O. Silva (2015) p. 95150B.

[65] C. Le Blanc, D. Papadopoulos, G. Chériaux, P. Georges, J. P. Zou, G. Mennerat, F. Druon, A. Pellegrina, P. Ramirez, F. Giambruno, A. Fréneaux, F. Leconte, D. Badarau, J. M. Boudenne, P. Audebert, D. Four- net, T. Valloton, C. Greverie, J. L. Paillard, J. L. Veray, M. Pina, P. Monot, P. Martin, F. Mathieu, J. P. Chambaret, and F. Amiranoff, "The Apollon-10P project: Design and Diagnostics Challenges," (2013), http://www.laserlab-europe. net/networking/scientific-and-technologicalexchanges/workshop-characterisation-2013-clf/ 6-1-laser-diagnostics-for-the-apollon-10pwfacility-catherine-le-blanc, accessed September 2016.

[66] A. Specka, "Electron acceleration at CILEXAPOLLON," (2014), http://polywww.in2p3.fr/ IMG/pdf/20140515-VisiteMEXTOdoiSan.pdf accessed September 2016.

[67] Y. I. Salamin, Phys. Rev. A 92, 063818 (2015).

[68] T. G. Blackburn, Plasma Phys. Control. Fusion 57, $075012(2015)$

[69] A. M. Fedotov, N. B. Narozhny, G. Mourou, and G. Korn, Phys. Rev. Lett. 105, 080402 (2010)

[70] N. V. Elkina, A. M. Fedotov, I. Y. Kostyukov, M. V. Legkov, N. B. Narozhny, E. N. Nerush, and H. Ruhl, Phys. Rev. Spec. Top. - Accel. Beams 14, 054401 (2011)

[71] J. D. Hunter, Comput. Sci. Eng. 9, 90 (2007).

[72] O. Tange, ;login USENIX Mag. 36, 42 (2011)

[73] J. L. Volakis and K. Sertel, Integral Equation Methods for Electromagnetics (SciTech Publishing, Raleigh, NC, 2012). 